\title{
On time-fractional Lotka-Volterra diffusion model
}

\author{
Mine Aylin Bayrak and Ali Demir \\ Department of Mathematics, University of Kocaeli, Kocaeli, Turkey
}

Received: 23 March 2019, Accepted: 30 June 2019

Published online: 22 September 2019.

\begin{abstract}
The interest of this paper to find the solution of time-fractional Lotka-Volterra diffusion problem by implementing the residual power series method (RPSM). The fractional derivative is described in the Caputo sense. The desired solution of the nonlinear equations are established in the form of rapidly convergent series whose components are computed by Matlab Software Package. The obtained results and graphical consequences show that the suggested method in this study is very efficient, effective and reliable for the solution of the time-fractional Lotka-Volterra equation.
\end{abstract}

Keywords: Fractional differential equation, Lotka-Volterra equation, Caputo derivative, residual power series method.

\section{Introduction}

The Lotka-Volterra diffusion equations which has many applications in ecology, chemistry, genetics, etc. have been widely analyzed in recent years. In homogeneous model there could be different cases which is tough to guess. In one case, the more diffusive species could ignore its competitor for a long time and in another case they eliminate the competitor gradually when they invade the whole region. The unexpected result could happen in competitive reaction-diffusion system. The general form of Lotka-Volterra diffusion equations for two species can be given in the following form:

$$
\begin{aligned}
& D_{t}^{\alpha} u=d_{1} \Delta_{x} u+u\left(r_{1}-a_{1} u-k_{1} v\right), \Omega \times(0,+\infty) \\
& D_{t}^{\alpha} v=d_{2} \Delta_{x} v+v\left(r_{2}-a_{2} v-k_{2} u\right), \Omega \times(0,+\infty)
\end{aligned}
$$

where $\Omega$ represents some spatial domain and $d_{1}, d_{2}, r_{1}, r_{2}, a_{1}, a_{2}, k_{1}, k_{2}$ are some positive constants. Without diffusion , this system can be written as an ODE system. Then, the system has to steady state $(u, v)=(0,1)$ and $(u, v)=(1,0)$ which are stable when $\frac{k_{1} r_{2}}{r_{1} a_{2}}>1$ and $\frac{k_{2} r_{1}}{r_{2} a_{1}}>1$, respectively [1-4].

In recent years, fractional calculus which has been considerable interest are used in bioengineering, thermodynamics, viscoelasticity, control theory, aerodynamics, electromagnetics, signal processing, chemistry, finance [5-11]. Various numerical methods have been applied and analyzed for differential equations with fractional order derivative of Riemann-Liouville or Caputo sense [10-16]. The RPSM was established as an powerful method for fuzzy differential equations [17]. It has been successfully applied in various fields [18-29]. The solution of problems by RPSM are obtained in the form of Maclaurin series.

\section{Preliminaries}

In this section, the fundamental definitions and various features for fractional calculus are shown [10,30-32]. 
Definition 1. The Riemann-Liouville fractional integral of order $\alpha(\alpha \geq 0)$ is given as [19,22]

$$
\begin{gathered}
J^{\alpha} f(x)=\frac{1}{\Gamma(\alpha)} \int_{0}^{x}(x-t)^{\alpha-1} f(t) d t, \alpha>0, x>0 \\
J^{0} f(x)=f(x)
\end{gathered}
$$

Definition 2. The Caputo fractional derivative with order $\alpha$ is given as [19,22]

$$
D^{\alpha} f(x)=J^{m-\alpha} D^{m} f(x)=\int_{0}^{x}(x-t)^{m-\alpha-1} \frac{d^{m}}{d t^{m}} f(t) d t, m-1<\alpha<m, x>0
$$

where $D^{m}$ represents the differential operator with order $m$.

Taking the Caputo derivative, we have

$$
\begin{gathered}
D^{\alpha} x^{\beta}=0, \quad \beta<\alpha \\
D^{\alpha} x^{\beta}=\frac{\Gamma(\beta+1)}{\Gamma(\beta+1-\alpha)} x^{\beta-\alpha}, \quad \beta \geq \alpha
\end{gathered}
$$

Definition 3. The Caputo's time fractional derivative of order $\alpha$ of $u(x, t)$ is defined as [19,22]

$D_{t}^{\alpha} u(x, t)=\left\{\begin{array}{cc}\frac{1}{\Gamma(m-\alpha)} \int_{0}^{t}(t-\xi)^{m-\alpha-1} \frac{\partial^{m} u(x, \xi)}{\partial t^{m}} d \xi, m-1<\alpha<m \\ \frac{\partial^{m} u(x, t)}{\partial t^{m}} \quad, \alpha=m \in N\end{array}\right.$

Theorem 1. Suppose that $u(x, t)$ has a multiple fractional power series representation at $t=t_{0}$ of the form

$$
u(x, t)=\sum_{m=0}^{\infty} f_{m}(x)\left(t-t_{0}\right)^{m \alpha}, \quad x \in I, \quad t_{0} \leq t \leq t_{0}+R
$$

If $D_{t}^{m \alpha} u(x, t), m=0,1,2, \ldots$ are continuous on $I \times\left(t_{0}, t_{0}+R\right)$, then $f_{m}(x)=\frac{D_{t}^{m \alpha} u\left(x, t_{0}\right)}{\Gamma(m \alpha+1)}$.

\section{Application RPSM to the time-fractional Lotka-Volterra diffusion problem}

We first consider the following one-dimensional Lotka-Volterra competition-diffusion problem:

$$
\begin{aligned}
& D_{t}^{\alpha} u=d_{1} D_{x x} u+u\left(r_{1}-a_{1} u-k_{1} v\right), R \times(0,+\infty) \\
& D_{t}^{\alpha} v=d_{2} D_{x x} v+v\left(r_{2}-a_{2} v-k_{2} u\right), R \times(0,+\infty)
\end{aligned}
$$

subject to initial condition

$$
\begin{gathered}
u(x, 0)=A_{1}(x), \\
v(x, 0)=B_{1}(x)
\end{gathered}
$$

where $d_{1}, d_{2}, r_{1}, r_{2}, a_{1}, a_{2}, k_{1}, k_{2}$ are positive constants with ecological meaning. The RPSM is applied to find out series solution for these equations with given initial conditions by replacing its fractional power series expansion with its truncated residual function. From each equation, a repetition formula for the determination of coefficients is supplied, while coefficients in fractional power series expansion can be calculated by repeatedly fractional differentiation of the 
truncated residual function [17-24]. The RPSM propose the solutions for Eq. (10)-(13) as a fractional power series at $t=0[17]$

$$
\begin{aligned}
& u(x, t)=\sum_{k=0}^{\infty} A_{k+1}(x) \frac{t^{k \alpha}}{\Gamma(k \alpha+1)}, \\
& v(x, t)=\sum_{k=0}^{\infty} B_{k+1}(x) \frac{t^{k \alpha}}{\Gamma(k \alpha+1)}
\end{aligned}
$$

where $x \in I, 0 \leq t<R$. To obtain the numerical values from this series, let $u_{m}(x, t)$ denotes the $m$-th truncated series of $u(x, t)$. That is

$$
\begin{aligned}
& u_{m}(x, t)=\sum_{k=0}^{m} A_{k+1}(x) \frac{t^{k \alpha}}{\Gamma(k \alpha+1)}, \\
& v_{m}(x, t)=\sum_{k=0}^{m} B_{k+1}(x) \frac{t^{k \alpha}}{\Gamma(k \alpha+1)} .
\end{aligned}
$$

By the condition at $t=0$, we have

$$
\begin{aligned}
& u_{0}(x, t)=f_{0}(x)=u(x, 0)=A_{1}(x), \\
& v_{0}(x, t)=g_{0}(x)=v(x, 0)=B_{1}(x)
\end{aligned}
$$

From Eqs.(14)-(15)

$$
\begin{aligned}
& u_{m}(x, t)=A_{1}(x)+\sum_{k=2}^{m} A_{k}(x) \frac{t^{k \alpha}}{\Gamma(k \alpha+1)}, 0<\alpha \leq 1, \\
& v_{m}(x, t)=B_{1}(x)+\sum_{k=2}^{m} B_{k}(x) \frac{t^{k \alpha}}{\Gamma(k \alpha+1)}, 0<\alpha \leq 1 .
\end{aligned}
$$

Define the residual function as for Eqs.(10)-(11)[35]

$$
\begin{gathered}
\operatorname{Resu}_{0}=D_{t}^{\alpha} u_{0}-d_{1} D_{x x} u_{0}+u_{0}\left(r_{1}-a_{1} u_{0}-k_{1} v_{0}\right) \\
\operatorname{Res}_{0}=D_{t}^{\alpha} v_{0}-d_{2} D_{x x} v_{0}+v_{0}\left(r_{2}-a_{2} v_{0}-k_{2} u_{0}\right) \\
\operatorname{Resu}_{m}=D_{t}^{\alpha} u_{m}-d_{1} D_{x x} u_{m}+u_{m}\left(r_{1}-a_{1} u_{m}-k_{1} v_{m}\right) \\
\operatorname{Resv}_{m}=D_{t}^{\alpha} v_{m}-d_{2} D_{x x} v_{m}+v_{m}\left(r_{2}-a_{2} v_{m}-k_{2} u_{m}\right)
\end{gathered}
$$

From [17-24], by making use of some results such as $\operatorname{Res}(x, t)=0$ and $D_{t}^{k \alpha} \operatorname{Res}_{m}(x, 0)=0, k=0,1,2, \ldots, m, m=1,2,3, \ldots$ are used to obtain the solution.

Substituting $u_{m}(x, t), v_{m}(x, t)$ into Eqs. (24)-(25), calculating the fractional derivative $D_{t}^{(m-1) \alpha}$ of $\operatorname{Res}(x, t)$ at $t=0$ and solving the following obtained algebraic system

$$
D_{t}^{(m-1) \alpha} \operatorname{Res}_{m}(x, 0)=0,0<\alpha \leq 1, m=1,2,3, \ldots
$$

the required coefficients $A_{k}(x), k=2,3, \ldots, m$ in Eq. (20) are determined. 
In order to determine $A_{2}(x)$ and $B_{2}(x)$, the 1 st residual function in Eqs. (24)-(25) can be written as follows:

$$
\begin{aligned}
& \operatorname{Resu}_{0}=D_{t}^{\alpha} u_{0}-d_{1} D_{x x} u_{0}+u_{0}\left(r_{1}-a_{1} u_{0}-k_{1} v_{0}\right) \\
& \operatorname{Res}_{0}=D_{t}^{\alpha} v_{0}-d_{2} D_{x x} v_{0}+v_{0}\left(r_{2}-a_{2} v_{0}-k_{2} u_{0}\right)
\end{aligned}
$$

where $u_{0}(x, t)=A_{1}(x)+A_{2}(x) \frac{t^{\alpha}}{\Gamma(1+\alpha)}$ and $v_{0}(x, t)=B_{1}(x)+B_{2}(x) \frac{t^{\alpha}}{\Gamma(1+\alpha)}$. Therefore,

$$
\begin{array}{r}
\operatorname{Resu}_{0}(x, t)=A_{2}-d_{1}\left(A_{1}^{\prime \prime}+A_{2}^{\prime \prime} \frac{t^{\alpha}}{\Gamma(1+\alpha)}\right)-r_{1}\left(A_{1}+A_{2} \frac{t^{\alpha}}{\Gamma(1+\alpha)}\right)+a_{1}\left(A_{1}+A_{2} \frac{t^{\alpha}}{\Gamma(1+\alpha)}\right)^{2} \\
+k_{1}\left(A_{1}+A_{2} \frac{t^{\alpha}}{\Gamma(1+\alpha)}\right)\left(B_{1}+B_{2} \frac{t^{\alpha}}{\Gamma(1+\alpha)}\right)
\end{array}
$$

$$
\begin{array}{r}
\operatorname{Resv}_{0}(x, t)=B_{2}-d_{2}\left(B_{1}^{\prime \prime}+B_{2}^{\prime \prime} \frac{t^{\alpha}}{\Gamma(1+\alpha)}\right)-r_{2}\left(B_{1}+B_{2} \frac{t^{\alpha}}{\Gamma(1+\alpha)}\right)+a_{2}\left(B_{1}+B_{2} \frac{t^{\alpha}}{\Gamma(1+\alpha)}\right)^{2} \\
+k_{2}\left(A_{1}+A_{2} \frac{t^{\alpha}}{\Gamma(1+\alpha)}\right)\left(B_{1}+B_{2} \frac{t^{\alpha}}{\Gamma(1+\alpha)}\right)
\end{array}
$$

From Eq.(26), it is concluded that $\operatorname{Resu}_{0}(x, 0)=0$ and $\operatorname{Resv}_{0}(x, 0)=0$, which leads to

$$
\begin{aligned}
& A_{2}(x)=d_{1} A_{1}^{\prime \prime}+r_{1} A_{1}-a_{1} A_{1}^{2}-k_{1} A_{1} B_{1} \\
& B_{2}(x)=d_{2} B_{1}^{\prime \prime}+r_{2} B_{1}-a_{2} B_{1}^{2}-k_{2} A_{1} B_{1}
\end{aligned}
$$

Similarly, to obtain $A_{3}(x)$ and $B_{3}(x)$, the 2 nd residual function in Eqs. (24)-(25) becomes

$$
\begin{gathered}
\operatorname{Resu}_{1}(x, t)=D_{t}^{\alpha} u_{1}-d_{1} D_{x x} u_{1}+u_{1}\left(r_{1}-a_{1} u_{1}-k_{1} v_{1}\right) \\
\operatorname{Res}_{1}(x, t)=D_{t}^{\alpha} v_{1}-d_{2} D_{x x} v_{1}+v_{1}\left(r_{2}-a_{2} v_{1}-k_{2} u_{1}\right)
\end{gathered}
$$

where $u_{1}(x, t)=A_{1}(x)+A_{2}(x) \frac{t^{\alpha}}{\Gamma(1+\alpha)}+A_{3}(x) \frac{t^{2 \alpha}}{\Gamma(1+2 \alpha)}$ and $v_{1}(x, t)=B_{1}(x)+B_{2}(x) \frac{t^{\alpha}}{\Gamma(1+\alpha)}+B_{3}(x) \frac{t^{2 \alpha}}{\Gamma(1+2 \alpha)}$. Therefore,

$$
\begin{aligned}
\operatorname{Resu}_{1}(x, t)= & \left.A_{2}+A_{3} \frac{t^{\alpha}}{\Gamma(1+\alpha)}\right)-d_{1}\left(A_{1}^{\prime \prime}+A_{2}^{\prime \prime} \frac{t^{\alpha}}{\Gamma(1+\alpha)}+A_{3}^{\prime \prime} \frac{t^{2 \alpha}}{\Gamma(1+2 \alpha)}\right) \\
& -r_{1}\left(A_{1}+A_{2} \frac{t^{\alpha}}{\Gamma(1+\alpha)}+A_{3} \frac{t^{2 \alpha}}{\Gamma(1+2 \alpha)}\right)+a_{1}\left(A_{1}+A_{2} \frac{t^{\alpha}}{\Gamma(1+\alpha)}+A_{3} \frac{t^{2 \alpha}}{\Gamma(1+2 \alpha)}\right)^{2} \\
& +k_{1}\left(A_{1}+A_{2} \frac{t^{\alpha}}{\Gamma(1+\alpha)}+A_{3} \frac{t^{2 \alpha}}{\Gamma(1+2 \alpha)}\right)\left(B_{1}+B_{2} \frac{t^{\alpha}}{\Gamma(1+\alpha)}+B_{3} \frac{t^{2 \alpha}}{\Gamma(1+2 \alpha)}\right) \\
\operatorname{Resv}_{1}(x, t)= & \left(B_{2}+B_{3} \frac{t^{\alpha}}{\Gamma(1+\alpha)}\right)-d_{2}\left(B_{1}^{\prime \prime}+B_{2}^{\prime \prime} \frac{t^{\alpha}}{\Gamma(1+\alpha)}+B_{3}^{\prime \prime} \frac{t^{2 \alpha}}{\Gamma(1+2 \alpha)}\right) \\
& -r_{2}\left(B_{1}+B_{2} \frac{t^{\alpha}}{\Gamma(1+\alpha)}+B_{3} \frac{t^{2 \alpha}}{\Gamma(1+2 \alpha)}\right)+a_{2}\left(B_{1}+B_{2} \frac{t^{\alpha}}{\Gamma(1+\alpha)}+B_{3} \frac{t^{2 \alpha}}{\Gamma(1+2 \alpha)}\right)^{2} \\
& +k_{2}\left(A_{1}+A_{2} \frac{t^{\alpha}}{\Gamma(1+\alpha)}+A_{3} \frac{t^{2 \alpha}}{\Gamma(1+2 \alpha)}\right)\left(B_{1}+B_{2} \frac{t^{\alpha}}{\Gamma(1+\alpha)}+B_{3} \frac{t^{2 \alpha}}{\Gamma(1+2 \alpha)}\right)
\end{aligned}
$$


The operator $D_{t}^{\alpha}$ is applied on both sides of Eqs.(34)-(35) as follows:

$$
\begin{aligned}
& D_{t}^{\alpha} \operatorname{Resu}_{1}(x, t)= A_{3}-d_{1}\left(A_{2}^{\prime \prime}+A_{3}^{\prime \prime} \frac{t^{\alpha}}{\Gamma(1+\alpha)}\right)-r_{1}\left(A_{2}+A_{3} \frac{t^{\alpha}}{\Gamma(1+\alpha)}\right) \\
&+2 a_{1}\left(A_{2}+A_{3} \frac{t^{\alpha}}{\Gamma(1+\alpha)}\right)\left(A_{1}+A_{2} \frac{t^{\alpha}}{\Gamma(1+\alpha)}+A_{3} \frac{t^{2 \alpha}}{\Gamma(1+2 \alpha)}\right) \\
&+k_{1}\left(A_{2}+A_{3} \frac{t^{\alpha}}{\Gamma(1+\alpha)}\right)\left(B_{1}+B_{2} \frac{t^{\alpha}}{\Gamma(1+\alpha)}+B_{3} \frac{t^{2 \alpha}}{\Gamma(1+2 \alpha)}\right) \\
&+k_{1}\left(A_{1}+A_{2} \frac{t^{\alpha}}{\Gamma(1+\alpha)}+A_{3} \frac{t^{2 \alpha}}{\Gamma(1+2 \alpha)}\right)\left(B_{2}+B_{3} \frac{t^{\alpha}}{\Gamma(1+\alpha)}\right) \\
& D_{t}^{\alpha} \operatorname{Resv}_{1}(x, t)= B_{3}-d_{2}\left(B_{2}^{\prime \prime}+B_{3}^{\prime \prime} \frac{t^{\alpha}}{\Gamma(1+\alpha)}\right)-r_{2}\left(B_{2}+B_{3} \frac{t^{\alpha}}{\Gamma(1+\alpha)}\right) \\
&+2 a_{2}\left(B_{2}+B_{3} \frac{t^{\alpha}}{\Gamma(1+\alpha)}\right)\left(B_{1}+B_{2} \frac{t^{\alpha}}{\Gamma(1+\alpha)}+B_{3} \frac{t^{2 \alpha}}{\Gamma(1+2 \alpha)}\right) \\
&+k_{2}\left(A_{2}+A_{3} \frac{t^{\alpha}}{\Gamma(1+\alpha)}\right)\left(B_{1}+B_{2} \frac{t^{\alpha}}{\Gamma(1+\alpha)}+B_{3} \frac{t^{2 \alpha}}{\Gamma(1+2 \alpha)}\right) \\
&+k_{2}\left(A_{1}+A_{2} \frac{t^{\alpha}}{\Gamma(1+\alpha)}+A_{3} \frac{t^{2 \alpha}}{\Gamma(1+2 \alpha)}\right)\left(B_{2}+B_{3} \frac{t^{\alpha}}{\Gamma(1+\alpha)}\right) \\
& \Gamma\left(1+x^{2 \alpha}\right. \\
& \Gamma(1+\alpha)
\end{aligned}
$$

From Eq. (26),

$$
\begin{aligned}
& A_{3}(x)=d_{1} A_{2}^{\prime \prime}+r_{1} A_{2}-2 a_{1} A_{1} A_{2}-k_{1} A_{2} B_{1}-k_{1} A_{1} B_{2} \\
& B_{3}(x)=d_{2} B_{2}^{\prime \prime}+r_{2} B_{2}-2 a_{2} B_{1} B_{2}-k_{2} A_{2} B_{1}-k_{2} A_{1} B_{2}
\end{aligned}
$$

The same manner is repeated as above, the following recurrence results is obtained

$$
\begin{aligned}
& A_{4}(x)=d_{1} A_{3}^{\prime \prime}+r_{1} A_{3}-2 a_{1} A_{2}^{2}-2 a_{1} A_{1} A_{3}-k_{1} A_{3} B_{1}-2 k_{1} A_{2} B_{2}-k_{1} A_{1} B_{3} \\
& B_{4}(x)=d_{2} B_{3}^{\prime \prime}+r_{2} B_{3}-2 a_{2} B_{2}^{2}-2 a_{2} B_{1} B_{3}-k_{2} A_{3} B_{1}-2 k_{2} A_{2} B_{2}-k_{2} A_{1} B_{3}
\end{aligned}
$$

and so on.

\section{Numerical results}

Example 1. We take $d_{1}=d_{2}=0.1, r_{1}=r_{2}=0.1, a_{1}=a_{2}=0.1, k_{1}=k_{2}=0.2$ and initial conditions $A_{1}(x)=1, B_{1}(x)=1-x^{2}$ in Eqs.(3)-(4).

Based on the obtained results, we conclude that RPS approximate solution is getting closer to the exact solution of time-fractional Lotka-Volterra diffusion problem as the order of fractional derivative $\alpha$ increases to one.It is clear from Figs. 1-2 that convergence of the approximate solution depend on the order of the fractional derivative.

In Tables 1-2, the approximate solutions $u_{k}(x, t), v_{k}(x, t), k=0,1,2,3$ are given for $\alpha=0.25,0.5,1$. These tables show that as the fractional derivative $\alpha$ is getting closer to 1 , approximate solution getting closer to the exact solution of time-fractional Lotka-Volterra diffusion problem. 


\begin{tabular}{|c|c|c|c|}
\hline$t$ & $\alpha=0.25$ & $\alpha=0.5$ & $\alpha=1$ \\
\hline 0 & 1.00000 & 1.00000 & 1.00000 \\
\hline 0.2 & 0.92888 & 0.94624 & 0.97274 \\
\hline 0.4 & 0.91550 & 0.93116 & 0.95042 \\
\hline 0.6 & 0.90480 & 0.91970 & 0.93223 \\
\hline 0.8 & 0.89527 & 0.90905 & 0.91735 \\
\hline 1 & 0.88641 & 0.89823 & 0.90498 \\
\hline
\end{tabular}

Table 1: The $u$ solution for Ex. 1 for several values $\alpha$ and $x=0.5$.

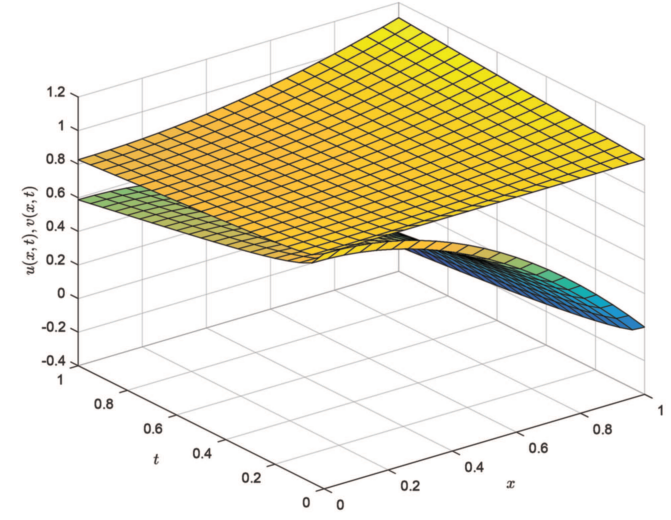

Fig. 1: The RPS solution for Ex.1 for $\alpha=0.5$.

\begin{tabular}{|c|c|c|c|}
\hline$t$ & $\alpha=0.25$ & $\alpha=0.5$ & $\alpha=1$ \\
\hline 0 & 1.00000 & 1.00000 & 1.00000 \\
\hline 0.2 & 0.03685 & 0.01989 & 0.00573 \\
\hline 0.4 & 0.04799 & 0.03241 & 0.01290 \\
\hline 0.6 & 0.05621 & 0.04372 & 0.02154 \\
\hline 0.8 & 0.06299 & 0.05442 & 0.03163 \\
\hline 1 & 0.06886 & 0.06472 & 0.04318 \\
\hline
\end{tabular}

Table 3: The $u$ solution for Ex. 2 for several values $\alpha$ and $x=0.5$.

\begin{tabular}{|c|c|c|c|}
\hline$t$ & $\alpha=0.25$ & $\alpha=0.5$ & $\alpha=1$ \\
\hline 0 & 0.75000 & 0.75000 & 0.75000 \\
\hline 0.2 & 0.52964 & 0.60076 & 0.68627 \\
\hline 0.4 & 0.48152 & 0.54185 & 0.62687 \\
\hline 0.6 & 0.44577 & 0.49413 & 0.57071 \\
\hline 0.8 & 0.41580 & 0.45075 & 0.51670 \\
\hline 1 & 0.38929 & 0.40926 & 0.46375 \\
\hline
\end{tabular}

Table 2: The $v$ solution for Ex. 1 for several values $\alpha$ and $x=0.5$.

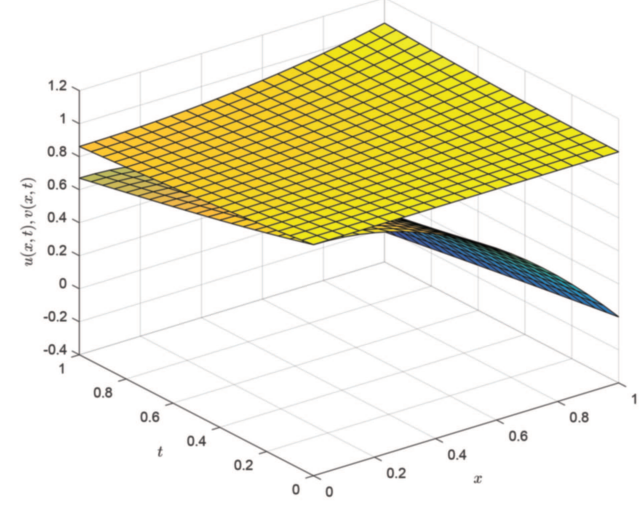

Fig. 2: The RPS solution for Ex.1 for $\alpha=1$.

\begin{tabular}{|c|c|c|c|}
\hline$t$ & $\alpha=0.25$ & $\alpha=0.5$ & $\alpha=1$ \\
\hline 0 & 0.75000 & 0.75000 & 0.75000 \\
\hline 0.2 & 0.60314 & 0.65555 & 0.71365 \\
\hline 0.4 & 0.56927 & 0.61268 & 0.67689 \\
\hline 0.6 & 0.54485 & 0.57731 & 0.63945 \\
\hline 0.8 & 0.52492 & 0.54545 & 0.60103 \\
\hline 1 & 0.50770 & 051559 & 0.56135 \\
\hline
\end{tabular}

Table 4: The $v$ solution for Ex. 2 for several values $\alpha$ and $x=0.5$.

Figs. 1-2, the approximate solutions $u(x, t), v(x, t)$ for $\alpha=0.5,1$ are plotted. It is clear from these figures that as the amount of $\alpha$ enlarges to one, the approximate solution getting closer to exact solution.

Example 2. Let $d_{1}=d_{2}=0.1, r_{1}=r_{2}=0.2, a_{1}=a_{2}=0.1, k_{1}=k_{2}=0.1$ and initial conditions $A_{1}(x)=1, B_{1}(x)=1-x^{2}$ in Eqs.(3)-(4).

Based on the obtained results, we conclude that RPS approximate solution is getting closer to the exact solution of time-fractional Lotka-Volterra diffusion problem as the order of fractional derivative $\alpha$ increases to one.It is clear from Figs. 1-2 that convergence of the approximate solution depend on the order of the fractional derivative.

In Tables 3-4, the approximate solutions $u_{k}(x, t), v_{k}(x, t), k=0,1,2,3$ are presented for $\alpha=0.25,0.5,1$. These tables show that as the fractional derivative $\alpha$ is getting closer to 1 , approximate solution getting closer to the exact solution of 


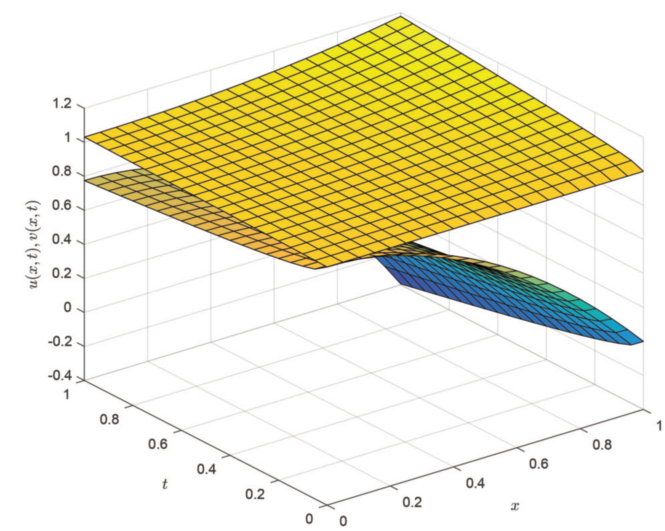

Fig. 3: The RPS solution for Ex.2 for $\alpha=0.5$.

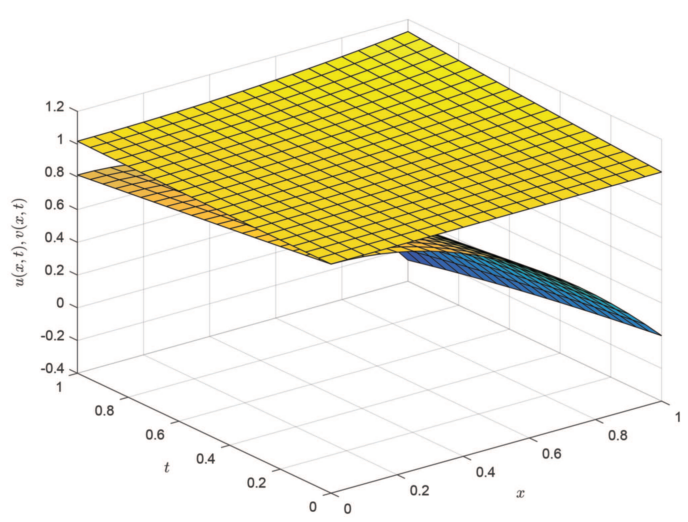

Fig. 4: The RPS solution for Ex. 2 for $\alpha=1$.

time-fractional Lotka-Volterra diffusion problem.

Figs. 3-4, the approximate solution $u(x, t), v(x, t)$ are drawn for $\alpha=0.5,1$. It is clear from these figures that as the amount of $\alpha$ enlarges to one, the approximate solution getting closer to exact solution.

\section{Competing interests}

The authors declare that they have no competing interests.

\section{Authors' contributions}

All authors have contributed to all parts of the article. All authors read and approved the final manuscript.

\section{References}

[1] K.J. Jesse, Modelling of a diffusive Lotka-Volterra system: the climate-induced shifting of tundra and forest realms in NorthAmerica, Ecol. Model. 123 (1999) 53-64.

[2] A.W. Leung, X. Hou,Y. Li, Exclusive traveling waves for competitive reaction-diffusion systems and their stabilities, J. Math. Anal.Appl. 338 (2008) 902-924.

[3] E.N. Dancer , D. Hilhorst ,M. Mimura , L.A. Peletier, Spatial segregation limit of a competition-diffusion system with Dirichlet boundary conditions, Nonlinear Anal. Real World. Appl. 5 (4) (2004) 645-665.

[4] L. Girardin , G. Nadin, Travelling Waves for diffusive and strongly competitive systems:Relative Motility and Invasion speed, arXiv:1503.06076v1.

[5] R.L. Bagley, P.J. Torvik, A theoretical basis for the application of fractional calculus to viscoelasticity, J. Rheol. 27 (1983) $201-210$.

[6] R.L. Bagley, P.J. Torvik, Fractional calculus a different approach to the analysis of viscoelastically damped structures, AIAA J. 21(5) (1983) 741-748.

[7] R.L. Bagley, P.J. Torvik, Fractional calculus in the transient analysis of viscoelastically damped structures, AIAA J. 23 (1985) 918-925.

[8] R.L. Magin, Fractional calculus in bioengineering, Crit. Rev. Biomed. Eng. 32 (2004) 1-104.

[9] D.A.Robinson, The use of control systems analysis in neurophysiology of eye movements, Ann. Rev. Neurosci. 4 (1981) $462-503$. [10] I. Podlubny, Fractional Differential equations, Academic Press, San Diego, 1999. 
[11] A.A. Kilbas,H.M. Srivastava, J.J. Trujillo, Theory and Applications of Fractional Differential Equations: North-Holland Mathematics Studies, vol. 204. Elsevier, Amsterdam, 2006.

[12] V. Daftardar-Gejji, A. Babakhani, Analysis of a system of fractional differential equations, J. Math. Anal. Appl. 293(2)(2004) 511-522.

[13] Z. Odibat, Approximations of fractional integrals and Caputo fractional derivatives, Appl.Math.Comput. 178(2)(2006) $527-533$.

[14] J. Sabatier, O.P. Agrawal, J.A.T. Machado JAT (eds), Advances in fractional calculus: theoretical developments and applications in physics and engineering, Dordrecht: Springer, 2007.

[15] S.G. Samko , A.A. Kilbas AA, O.I. Marichev, Fractional integrals and derivatives: theory and applications, Amsterdam: Gordon and Breach, 1993.

[16] D. Baleanu , K. Diethelm , E. Scalas, et al, Fractional: calculus models and numerical methods (complexity, nonlinearity, and chaos), Boston, MA: World Scientific, 2012.

[17] O. Abu Arqub, Series solution of fuzzy differential equations under strongly generalized differentiability, J. Adv. Res. Appl. Math. 5 (2013) 31-52.

[18] O. Abu Arqub, A. El-Ajou, A. Bataineh , et al, A representation of the exact solution of generalized Lane Emden equations using a new analytical method, Abstr. Appl. Anal. 2013(2013) 378-593.

[19] A. El-Ajou ,O. Abu Arqub , Z. Al Zhour, et al, New results on fractional power series: theories and applications, Entropy 16 (2013) 5305-5323.

[20] O. Abu Arqub , A. El-Ajou ,Z. Al Zhour , et al, Multiple solutions of nonlinear boundary value problems of fractional order: a new analytic iterative technique, Entropy 16 (2014) 471-493.

[21] O. Abu Arqub , A. El-Ajou ,S. Momani , Constructing and predicting solitary pattern solutions for nonlinear time-fractional dispersive partial differential equations, J. Comput. Phys. 293 (2015) 385-399.

[22] A. El-Ajou, O. Abu Arqub , S. Momani , Approximate analytical solution of the nonlinear fractional KdV-Burgers equation: a new iterative algorithm, J. Comput. Phys. 293 (2015) 81-95.

[23] M. Alquran , K. Al-Khaled , J. Chattopadhyay, Analytical solutions of fractional population diffusion model: residual power series, Nonlin. Stud. 22 (2015) 31-39.

[24] A. El-Ajou, O. Abu Arqub , S. Momani, et al, A novel expansion iterative method for solving linear partial differential equations of fractional order, Appl. Math. Comput. 257 (2015) 119-133.

[25] R.A. Fisher, The wave of advance of advantageous genes, Annu. Eugen. 7 (1937) 355-369.

[26] I.C. Sungu , H. Demir, New approach and solution technique to solve time fractional nonlinear reaction diffusion equations, Math. Probl. Eng. 2015(2015) 457013.

[27] S.Z. Rida , A.M.A. El-Sayed, A.A.M. Arafa, On the solutions of time-fractional reaction-diffusion equations, Commun. Nonlinear Sci. 15 (2010) 3847-3854.

[28] N.A. Khan , F.Ayaz , L. Jin , et al, On approximate solutions for the time-fractional reaction-diffusion equation of Fisher type, Int. J. Phys. Sci. 6 (2011) 2483-2496.

[29] V.K. Baranwal, R.K. Pandey, M.P. Tripathi , et al, An analytic algorithm for time fractional nonlinear reaction-diffusion equation based on a new iterative method, Commun. Nonlinear Sci. 17 (2012) 3906-3921.

[30] M. Merdan,Solutions of time-fractional reaction-diffusion equation with modified Riemann-Liouville derivative, Int. J. Phys. Sci. 7 (2012) 2317-2326.

[31] K.B. Oldham, J. Spanier, The Fractional Calculus: Theory and Application of Differentiation and Integration to Arbitrary order, Academic Press, California, 1974.

[32] K. Miller, B. Ross, An Introduction to the Fractional Calculus and Fractional Differential Equations, Wiley, New York, 1993.

[33] S.S. Ray, Analytical solution for the space fractional diffusion equation by two-step Adomian decomposition method, Commun. Nonlinear Sci. Numer. Simul. 14 (2009) 1130-1295.

[34] R. Magin, X. Feng, D. Baleanu, Solving fractional order Bloch equation, Concept magnetic Res. 34A (2009) 16-23.

[35] M. Mohamed Al Qurashi , Z. Korpinar , D. Baleanu, M. Inc , A new iterative algorithm on the time-fractional Fisher equation: Residual power series method, Adv. in Mech. Eng. 9(9)(2017) 1-8. 